\title{
Pulsar VLBI Experiment with Kashima(Japan)-Kalyazin(Russia) Baseline
}

\author{
M. Sekido, M.Imae, Y. Hanado, Y. Takahashi, Y. Koyama \\ Communications Research Laboratory (CRL), Japan. \\ Y.P. Ilyasov, A.E. Rodin, A.E. Avramenko, V.V. Oreshko \\ Astro Space Center of P.N.Lebedev Physical Institute, Russia.
}

\section{B.A. Poperechenko}

\author{
Special Research Bureau of the Moscow Power Engineering Institute, \\ Russia.
}

\section{Introduction}

Interferometric measurement of a pulsar is one of the most effective techniques to measure accurate source position. Precise measurement of pulsar position will give us the information of proper motion and parallax. Interferometric measurements on pulsars have been carried out, but the number of the pulsars whose position were measured is still smaller than the total number of pulsars. Because newly discovered pulsars are rapidly increasing due to recent intensive systematic surveys. We have started measuring their position by using VLBI between Kashima and Kalyazin. This report shows the results of the first experiment.

\section{Observation}

This Pulsar VLBI observation program is based on collaboration between the Communications Research Laboratory(CRL) and the Lebedev Physical Institute. Our first VLBI observation was performed on 14 March 1995. We used a $34 \mathrm{~m}$ antenna at Kashima and $64 \mathrm{~m}$ antenna at Kalyazin, which is about $150 \mathrm{~km}$ north part of Moscow. The baseline length is about $7000 \mathrm{~km}$ and minimum fringe spacing is 7 mas at $1.4 \mathrm{GHz}$. The system temperature and efficiency of the $34 \mathrm{~m}$ antenna and the $64 \mathrm{~m}$ antenna are about $40 \mathrm{~K}$ and 0.6 respectively for both antennas. The employed VLBI data acquisition system is Japanese K4 system which is compatible with the Mark -III mode C.

\section{Correlation and Analysis}

Correlation processing was performed by a $\mathrm{K} 3$ correlator, which was developed by CRL. A gating function was available on the correlator, but we couldn't use it due to some problems in processing gated data. After ordinal cross correlation processing, the result was stored in the Mk-III database and analyzed with software 'SOLVE'. SOLVE is a program developed by NASA for baseline and source 


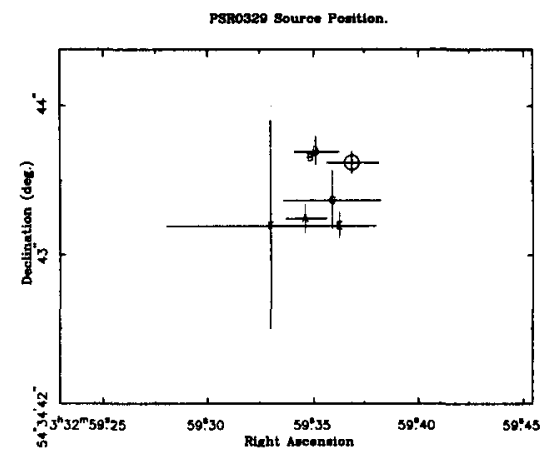

Figure 1. Estimated position of PSR0329+54. Our result is $\bigcirc$ and others are as follows: A: Taylor et al.(1993), B: VLBI by Bartel et al. (1985), C: $35 \mathrm{~km}$-Interferometer by Backer and Sramek (1981), D: VLA by Formalont et al. (1984), E: Pulse-Time-of-Arrival (PTA) measurement by Downs and Reichely(1983), F: PTA measurement by Helfand et al.(1980). Transformation between the deferent coordinate system is not applied in this figure.

position analysis with group delay. The estimated position of PSR0329+54 is

$$
\text { PSR0329+54: } \begin{array}{cc}
\alpha: & 3^{h} 32^{m} 59^{s} .369 \pm 0.01 \\
\delta: & 54^{\circ} 34^{\prime} 43^{\prime \prime} .627 \pm 0.08
\end{array}
$$

We are now developing a new correlator with gating function to improve the signal to noise ratio. Our observations will be continued for a few years to detect the proper motion.

Acknowledgments. We thanks to the staff of Kalyazin $64 \mathrm{~m}$ antenna operation team, especially V. A. Agafonov, A. N. Fatiev. and M. A. Dolgov. Also V. V. Oreshko, V. Sternenko, and A. V. Serov who helped us for receiver and atomic standard. Many thanks to members of CRL Radio astronomy applications section for their support of this experiment.

\section{References}

Backer,D. C., Sramek, R. A., 1981, In Pulsars, IAU Symposium No. 95, edited by W. Sieber and R. Wielebinski (Reidel, Derdriecht), 205.

Bartel,N., Ratner, M. I., Shapiro,I. I., Cappallo, R. J. Rogers, A. E. E., Whitney, A. R., 1985. ApJ, 90, 318

Downs,G. S., Reichley, P. E. 1983, ApJS, 53, 169.

Formalont, E. B., Gross, W. M., Lyne, A. G., and Manchester, R. N. 1984, MNRAS210, 113.

Helfand,D. J., Taylor, J. H., Backus, P. R., and Cordes, J. M. 1980, ApJ, 237, 206

Taylor,J. H., Manchester, and R. N., Lyne, A. G. 1993, ApJS, 88, 529 